\title{
THE POWER OF THE TESTS OF ROBINSON (1994) IN THE CONTEXT OF FRACTIONALLY INTEGRATED MOVING AVERAGE MODELS
}

\author{
Luis A. Gil-Alana* \\ Humboldt Universität zu Berlin, Institut für Statistik und Ökonometrie, Berlin, Germany \\ University of Navarre, Department of Economics, Pamplona, Spain
}

\begin{abstract}
We examine in this article the power of the tests of Robinson (1994) for testing I(d) statistical models in the presence of moving average (MA) disturbances. The results show that the tests behave relatively well if we correctly assume that the disturbances are MA. However, assuming white noise or autoregressive disturbances, the power of the tests against one-sided alternatives is very low.
\end{abstract}

JEL Classification: C12; C15.

Keywords: Fractional integration; Monte Carlo simulations.

Correspondence author: Luis A. Gil-Alana

Humboldt Universität zu Berlin

Institut für Statistik und Ökonometrie

Spandauer Str. 1

D-10178 Berlin, GERMANY

Phone: $\quad 00493020935908$

Fax: $\quad 00493020935712$

Email: $\quad$ alana@wiwi.hu-berlin.de

\footnotetext{
* Financial support from Macroeconomic Modelling and Policy Analysis in a Changing World Grant No. "L116251013" is gratefully acknowledged. Also, the author also acknowledges financial support from the Deutsche Forschungsgemeinschaft, SFB 373.
} 


\section{Introduction}

For the purpose of the present paper, we define an $\mathrm{I}(0)$ process as a covariance stationary process with spectral density function which is positive and finite at the zero frequency. In this context, we say that $x_{t}, t=0, \pm 1, \ldots$, is $I(d)$ if

$$
(1-L)^{d} x_{t}=u_{t}, \quad t=1,2, \ldots
$$

where $u_{t}$ is $I(0)$ and where $d$ can be any real number. If $d=0, x_{t}=u_{t}$, and a 'weakly' autocorrelated process is allowed for. However, if $\mathrm{d}>0, \mathrm{x}_{\mathrm{t}}$ is said to be a long memory process, also called strong dependent because of the strong association between observations widely separated in time. This type of model was introduced by Granger and Joyeux (1980), Granger (1980, 1981) and Hosking (1981) and were justified theoretically in terms of aggregation by Robinson (1978) and Granger (1980).

Robinson (1994) proposed Lagrange Multiplier (LM) tests for testing I(d) statistical models like (1) and most of the empirical applications based on his tests (eg., Gil-Alana and Robinson, 1997, Gil-Alana, 2000a,b) are based on disturbances which are white noise or autoregressions. In this paper we want to investigate if the tests of Robinson (1994) have power in the presence of moving average (MA) disturbances. The outline of the article is as follows: Section 2 briefly describes Robinson's (1994) tests. In Section 3, several Monte Carlo experiments are conducted to examine the size and the power of the tests when the true model contains MA components. Finally, Section 4 concludes.

\section{The tests of Robinson (1994)}

Let's suppose that $\left\{\mathrm{x}_{\mathrm{t}}, \mathrm{t}=1,2, \ldots, \mathrm{T}\right\}$ is the time series we observe. In general, we want to test the null hypothesis:

$$
H_{o}: d=d_{o},
$$


in (1) for any real value $d_{o}$. We assume that $u_{t}$ has parametric spectral density $f$, which is a given function of frequency $\lambda$ and of unknown parameters,

$$
f\left(\lambda ; \sigma^{2} ; \tau\right)=\frac{\sigma^{2}}{2 \pi} g(\lambda ; \tau), \quad-\pi<\lambda \leq \pi
$$

where the scalar $\sigma^{2}$ and the (qx1) vector $\tau$ are unknown but $g$ is assumed to be known. For example, in the AR case, if $\sigma^{2}=\mathrm{V}\left(\varepsilon_{\mathrm{t}}\right)$, we have $\mathrm{g}(\lambda ; \tau)=\left|\phi\left(\mathrm{e}^{\mathrm{i} \lambda}\right)\right|^{-2}$, where $\phi$ is the AR polynomial, so that the AR coefficients are functions of $\tau$. Specifically, the test statistic proposed by Robinson (1994) is given by:

$$
\begin{gathered}
\hat{r}=\left(\frac{T}{\hat{A}}\right)^{1 / 2} \frac{\hat{a}}{\hat{\sigma}^{2}} \\
\hat{a}=\frac{-2 \pi}{T} \sum_{j=1}^{T-1} \psi\left(\lambda_{j}\right) g\left(\lambda_{j} ; \hat{\tau}\right)^{-1} I\left(\lambda_{j}\right) ; \quad \hat{\sigma}^{2}=\frac{2 \pi}{T} \sum_{j=1}^{T-1} g\left(\lambda_{j} ; \hat{\tau}\right)^{-1} I\left(\lambda_{j}\right) ; \\
\hat{A}=\frac{2}{T}\left(\sum_{j=1}^{T-1} \psi\left(\lambda_{j}\right)^{2}-\sum_{j=1}^{T-1} \psi\left(\lambda_{j}\right) \varepsilon\left(\lambda_{j}\right)^{\prime} \times\left(\sum_{j=1}^{T-1} \varepsilon\left(\lambda_{j}\right) \varepsilon\left(\lambda_{j}\right)^{\prime}\right)^{-1} \times \sum_{j=1}^{T-1} \varepsilon\left(\lambda_{j}\right) \psi\left(\lambda_{j}\right)\right) \\
\psi\left(\lambda_{j}\right)=\log \left|2 \sin \frac{\lambda_{j}}{2}\right| ; \quad \hat{\varepsilon}\left(\lambda_{j}\right)=\frac{\partial}{\partial \tau} \log g\left(\lambda_{j} ; \hat{\tau}\right),
\end{gathered}
$$

$\mathrm{I}\left(\lambda_{\mathrm{j}}\right)$ is the periodogram of $\hat{u}_{t}=(1-L)^{d_{o}} x_{t}$, evaluated at $\lambda_{\mathrm{j}}=2 \pi \mathrm{j} / \mathrm{T}$, and $\hat{\tau}=\arg \min _{\tau \in T^{*}} \sigma^{2}(\tau)$ with $\mathrm{T}^{*}$ as a suitable subset of the $\mathrm{R}^{\mathrm{q}}$ Euclidean space.

Robinson (1994) showed that under certain regularity conditions,

$$
\hat{r} \rightarrow_{d} N(0,1), \quad \text { as } \quad T \rightarrow \infty
$$

and also the Pitman efficiency property against local departures from the null. Thus, a test of (2) against $\mathrm{H}_{\mathrm{a}}: \mathrm{d}>\mathrm{d}_{\mathrm{o}}\left(\mathrm{d}<\mathrm{d}_{\mathrm{o}}\right)$ will reject $\mathrm{H}_{\mathrm{o}}$ if $\hat{r}>\mathrm{z}_{\alpha}\left(\hat{r}<-\mathrm{z}_{\alpha}\right)$, where the probability that a normal standard variate exceeds $z_{\alpha}$ is $\alpha$.

Gil-Alana (2000a) studied the size and the power properties of Robinson's (1994) tests in finite samples, computing finite-sample critical values for the cases of white noise and AR 
disturbances. In the following section, we examine if the tests of Robinson (1994) also have power against models which incorporate MA components.

\section{The power of Robinson's (1994) tests with MA components}

We look at the size and the power properties of Robinson's (1994) tests when the true model is given by:

$$
(1-L) x_{t}=u_{t} ; \quad u_{t}=\varepsilon_{t}+\theta \varepsilon_{t-1},
$$

with $\theta=0.25,0.5$ and 0.75 and white noise $\varepsilon_{t}$. Table 1 reports the rejection frequencies of $\hat{r}$ in (3) when the alternatives are of form as in (1) with $d=0,(0.25), 2$ and we perform the test statistics based on white noise, $\operatorname{AR}(1)$ and $\mathrm{MA}(1)$ disturbances. Thus, the rejection frequencies corresponding to $d=1$ with $\mathrm{MA}(1) \mathrm{u}_{\mathrm{t}}$ will indicate the sizes of the tests. We generate Gaussian series generated by the routines GASDEV and RAN3 of Press, Flannery, Teukolsky and Vetterling (1986), with 10,000 replications of each case. The sample sizes are 40, 80 and 120 observations and in all cases the nominal size is $5 \%$.

\section{(Table 1 about here)}

Starting with the size, we see in this table that it is too small against alternatives of form: $\mathrm{H}_{\mathrm{a}}: \mathrm{d}>\mathrm{d}_{\mathrm{o}}$, but too large against $\mathrm{H}_{\mathrm{a}}: \mathrm{d}<\mathrm{d}_{\mathrm{o}}$, though it considerably improves as we increase the sample size. We also observe that the values are closer to the nominal value of $5 \%$ as we increase the value of $\theta$. This asymmetry in size is also associated with some different rejection frequencies depending on the alternatives and thus, higher rejection probabilities are observed when the alternatives are of form $\mathrm{H}_{\mathrm{a}}$ : $\mathrm{d}<\mathrm{d}_{\mathrm{o}}$. If we perform the tests supposing that the disturbances are white noise, we see that the rejection frequencies are relatively high for all sample sizes against $\mathrm{H}_{\mathrm{a}}$ : $\mathrm{d}>\mathrm{d}_{\mathrm{o}}$ but small values are obtained if $\mathrm{d}$ is equal to or slightly higher than one. This is observed for all sample sizes and all values of $\theta$. We see, for example, that if $\mathrm{T}$ $=120$, the rejection frequencies when $\mathrm{d}=1$ against $\mathrm{d}>1$ are $0.04,0.02$ and 0.01 for $\theta=0.25$, 
0.50 and 0.75 respectively. Assuming AR(1) disturbances, the rejection frequencies behave in the opposite way, obtaining higher values if $d<d_{o}$ than if $d>d_{0}$. In the latter case, the rejection probabilities never exceed 0.5 for values of $\mathrm{d}$ between 0 and 1 even if $\mathrm{T}=120$.

\section{Conclusion}

We have shown in this article that the tests of Robinson (1994) for testing I(d) statistical models have very low power when we misspecify the disturbances of the process. Thus, for example, if the true model contains MA disturbances and we perform the tests with white noise or AR(1) $u_{t}$, the rejection frequencies of the tests are very low against one-sided alternatives. However, correctly assuming MA disturbances, the size and the power of the tests are relatively good, especially if the sample size is large. Thus, we may conclude by saying that before performing the tests of Robinson (1994) for testing I(d) statistical models, we should take some care about the way of modelling the $\mathrm{I}(0)$ disturbances underlying the process. 


\section{References}

Gil-Alana, L.A., 2000a, Mean reversion in the real exchange rates, forthcoming in Economics Letters.

Gil-Alana, L.A., 2000b, Testing stochastic cycles in macroeconomic time series, forthcoming in Journal of Time Series Analysis.

Gil-Alana, L.A., 2000c, Evaluation of Robinson's (994) tests in finite samples, forthcoming in Journal of Statistical Computation and Simulation.

Gil-Alana, L.A. and P.M. Robinson, 1997, Testing of unit roots and other nonstationary hypotheses in macroeconomic time series, Journal of Econometrics 80, 241-268.

Granger, C.W.J., 1980, Long memory relationships and the aggregation of dynamic models, Journal of Econometrics 14, 227-238.

Granger, C.W.J., 1981, Some properties of time series data and their use in econometric model specification, Journal of Econometrics 16, 121-130.

Granger, C.W.J. and R. Joyeux, 1980, An introduction to long memory time series and fractionally differencing, Journal of Time Series Analysis 1, 15-29.

Hosking, J.R.M., 1981, Modelling persistence in hydrological time series using fractional differencing, Water Resources Research 20, 1898-1908.

Press, W.H., Flannery, B.P., Teukolsky, S.A. and Vetterling, W.T., 1986, Numerical recipes: The art of scientific computing, Cambridge University Press, Cambridge.

Robinson, P.M., 1978, Statistical inference for a random coefficient autoregressive model, Scandinavian Journal of Statistics 5, 163-168.

Robinson, P.M., 1994, Efficient tests of nonstationary hypotheses, Journal of the American Statistical Association 89, 1420-1437. 


\begin{tabular}{|c|c|c|c|c|c|c|c|c|c|c|c|c|}
\hline \multicolumn{13}{|c|}{ TABLE 1} \\
\hline \multicolumn{13}{|c|}{ Rejection frequencies of the tests of Robinson (1994) with MA disturbances } \\
\hline \multicolumn{5}{|c|}{ True model: } & \multicolumn{5}{|c|}{$(1-\mathrm{L}) \mathrm{y}_{\mathrm{t}}=\mathrm{u}_{\mathrm{t}} ; \quad \mathrm{u}_{\mathrm{t}}=\varepsilon_{\mathrm{t}}+\theta \varepsilon_{\mathrm{t}-1}$. } & & & \\
\hline \multicolumn{5}{|c|}{ Alternatives: } & \multicolumn{3}{|c|}{$(1-L)^{d_{o}} y_{t}=u_{t}$} & \multicolumn{3}{|c|}{$\mathrm{u}_{\mathrm{t}} \sim \mathrm{I}(0)$} & & \\
\hline \multirow{11}{*}{$\mathrm{T}=40$} & & & \multicolumn{5}{|c|}{$\mathrm{d}>\mathrm{d}_{\mathrm{o}}$} & \multicolumn{5}{|c|}{$\mathrm{d}<\mathrm{d}_{\mathrm{o}}$} \\
\hline & & $\mathrm{u}_{\mathrm{t}} / \mathrm{d}_{\mathrm{o}}$ & 0.00 & 0.25 & 0.50 & 0.75 & 1.00 & 1.00 & 1.25 & 1.50 & 1.75 & 2.00 \\
\hline & \multirow{3}{*}{$\theta=.25$} & W.N. & .998 & .993 & .939 & .671 & .174 & .010 & .139 & .574 & .913 & .990 \\
\hline & & $\operatorname{AR}(1)$ & .143 & .030 & .002 & .001 & .002 & .694 & .656 & .782 & .903 & .964 \\
\hline & & MA(1) & .971 & .872 & .563 & .171 & 016 & .241 & .690 & .972 & .999 & 1000 \\
\hline & \multirow{3}{*}{$\theta=.50$} & W.N. & .999 & .996 & .975 & .838 & .407 & .005 & .012 & .151 & .541 & .873 \\
\hline & & $\operatorname{AR}(1)$ & .250 & .121 & .028 & .004 & .006 & .851 & .844 & .940 & .956 & .982 \\
\hline & & $\mathrm{MA}(1)$ & .981 & .915 & .657 & .230 & .020 & .177 & .538 & .888 & .992 & .999 \\
\hline & \multirow{3}{*}{$\theta=.75$} & W.N. & .999 & .998 & .985 & .894 & .547 & .001 & .015 & .344 & .231 & .597 \\
\hline & & $\mathrm{AR}(1)$ & .298 & .208 & .084 & .023 & .004 & .793 & .910 & .960 & .987 & .996 \\
\hline & & MA(1) & .984 & .941 & .731 & .279 & .023 & .155 & .541 & .880 & .984 & .999 \\
\hline \multirow{11}{*}{$\mathrm{T}=80$} & & & \multicolumn{5}{|c|}{$\mathrm{d}>\mathrm{d}_{\mathrm{o}}$} & \multicolumn{5}{|c|}{$\mathrm{d}<\mathrm{d}_{\mathrm{o}}$} \\
\hline & & $\mathrm{u}_{\mathrm{t}} / \mathrm{d}_{\mathrm{o}}$ & 0.00 & 0.25 & \begin{tabular}{|l|}
0.50 \\
\end{tabular} & 0.75 & 1.00 & 1.00 & 1.25 & 1.50 & 1.75 & 2.00 \\
\hline & \multirow{3}{*}{$\theta=.25$} & W.N. & 1.000 & 1.000 & .999 & .950 & .348 & .001 & .155 & .841 & .996 & 1.000 \\
\hline & & $\mathrm{AR}(1)$ & .242 & .020 & .002 & .005 & .004 & .499 & .687 & .929 & .992 & .998 \\
\hline & & MA(1) & 1.000 & .998 & .928 & .437 & .021 & .140 & .753 & .997 & 1.000 & 1.000 \\
\hline & \multirow{3}{*}{$\theta=.50$} & W.N. & 1.000 & 1.000 & 1.000 & .990 & .729 & .001 & .004 & .236 & .845 & .995 \\
\hline & & $\operatorname{AR}(1)$ & .406 & .106 & .031 & .008 & .001 & .693 & .872 & .984 & .998 & .999 \\
\hline & & MA(1) & 1.000 & .999 & .961 & .535 & .024 & .122 & .682 & .984 & .999 & 1.000 \\
\hline & \multirow{3}{*}{$\theta=.75$} & W.N. & 1.000 & 1.000 & 1.000 & .996 & .866 & .001 & .009 & .037 & .451 & .911 \\
\hline & & $\operatorname{AR}(1)$ & .461 & .323 & .123 & .055 & .002 & .603 & .924 & .997 & 1.000 & 1.000 \\
\hline & & MA(1) & 1.000 & .999 & .977 & .612 & .026 & .110 & .752 & .992 & 1.000 & 1.000 \\
\hline \multirow{11}{*}{$\mathrm{T}=120$} & & & \multicolumn{5}{|c|}{$\mathrm{d}>\mathrm{d}_{\mathrm{o}}$} & \multicolumn{5}{|c|}{$\mathrm{d}<\mathrm{d}_{\mathrm{o}}$} \\
\hline & & $\mathrm{u}_{\mathrm{t}} / \mathrm{d}_{\mathrm{o}}$ & 0.00 & 0.25 & 0.50 & 0.75 & 1.00 & 1.00 & 1.25 & 1.50 & 1.75 & 2.00 \\
\hline & \multirow{3}{*}{$\theta=.25$} & W.N. & 1.000 & 1.00 & 1.000 & .994 & .496 & .004 & .187 & .954 & 1.000 & 1.000 \\
\hline & & $\mathrm{AR}(1)$ & .312 & .017 & .001 & .020 & .005 & .411 & .774 & .985 & .999 & 1.000 \\
\hline & & MA(1) & 1.000 & .999 & .990 & .647 & .026 & .112 & .828 & .999 & 1.000 & 1.000 \\
\hline & \multirow{3}{*}{$\theta=.50$} & W.N. & 1.000 & 1.000 & 1.000 & .999 & .890 & .001 & .002 & .343 & .964 & .999 \\
\hline & & $\operatorname{AR}(1)$ & .500 & .199 & .041 & .023 & .002 & .592 & .930 & .998 & 1.000 & 1.000 \\
\hline & & MA(1) & 1.000 & 1.000 & .997 & .741 & .028 & .104 & .818 & .998 & 1.000 & 1.000 \\
\hline & & W.N. & 1.000 & 1.000 & 1.000 & 1.000 & .969 & .002 & .001 & .048 & .663 & .987 \\
\hline & $\theta=.75$ & $\mathrm{AR}(1)$ & .556 & .406 & .169 & .115 & .004 & .469 & .961 & 1.000 & 1.000 & 1.000 \\
\hline & & MA(1) & 1.000 & 1.000 & .999 & .807 & .029 & .094 & .887 & .999 & 1.000 & 1.000 \\
\hline
\end{tabular}

